[0212-7199 (2007) 24: 5; pp 249-252] ANALES DE MEDICINA INTERNA Copyright (C) 2007 ARAN EDICIONES, S.L.

AN. MED INTERNA (Madrid) Vol. 24, N. ${ }^{\circ}$, pp. 249-252, 2007

\title{
El placebo en la práctica y en la investigación clínica
}

\author{
S. G. TEMPONE PÉREZ
}

Servicio de Anestesiología. Hospital Italiano de Buenos Aires. Argentina

THE PLACEBO IN MEDICAL PRACTICE AND RESEARCH

\section{RESUMEN}

El placebo es una herramienta terapéutica conocida desde la antigüedad, cuyo uso se realiza en la práctica y la en investigación clínica. En el presente trabajo he definido el concepto y resumido las pautas de utilización internacional en protocolos clínicos.

PALABRAS CLAVE: Placebo. Investigación clínica. Ética.
ABSTRACT

The placebo is a therapeutics tool well known since the antiquity. It is used in clinical practice and research. In this article I have define the concept and resume the guidelines for its use in clinical research.

Tempone Pérez SG. El placebo en la práctica y en la investigación clínica. An Med Interna (Madrid) 2007; 24: $249-252$.

Si bien el placebo es conocido desde hace mucho tiempo, su efecto, su utilidad y su indicación siguen siendo temas controvertidos. Como lo expresa el Dr. Polito la pregunta que surge en el médico frente al efecto placebo observado en su paciente es: ¿qué se pone en marcha cuando un paciente recibe un placebo? Y la respuesta inevitable sería ¿quién lo sabe? (1).

Lilienfeld comenta que el primer ensayo controlado con placebo del que se tiene conocimiento, fue probablemente realizado en el año 1931, cuando una droga conocida como sanocrysin se comparó con agua destilada en el tratamiento de la tuberculosis (2). Desde entonces el placebo se ha vinculado al tratamiento de algunas dolencias como asma, dolor y ansiedad, también se lo ha vinculado con la medicina alternativa. El placebo es el tratamiento patrón ("gold estándard") contra el cual se comparan los tratamientos de los ensayos clínicos. El objetivo es discriminar acciones farmacológicas de las drogas de otros factores psicológicos o físicos que puedan modificar los resultados observados. Vhrovac comenta que aun hoy en día los placebos siguen siendo una herramienta a tener en cuenta a pesar de la existencia de medicamentos específi$\cos$, y agrega que a veces en forma inadvertida los medicamentos son utilizados como placebos (3). Sin embargo, como nos recuerda Pérgola, haciendo referencia a Balint: “El médi- co es medicina", el médico debe tener presente que con su sola presencia tiene un efecto placebo incorporado en su investidura (4).

El efecto placebo, según el Dr Polito, tiene su origen en una palabra latina que significa "complaceré", haciendo referencia al médico que complace a su paciente con un tratamiento para alentar una esperanza. De esta forma en la indicación de un placebo se da un interjuego de expectativas: el médico espera que el paciente logre un beneficio clínico, y el paciente a su vez espera también una mejoría1.

\section{DEFINICIONES: EFECTO PLACEBO Y PLACEBO}

La palabra placebo se usaba en la Edad Media para designar los lamentos que proferían las plañideras profesionales, mujeres a las que se les pagaba por ir a llorar al funeral de alguna persona. En el siglo XVIII, el término fue definido en un diccionario médico como medicamento común y, en una edición posterior, como "algo" que simula ser un medicamento.

En la literatura se encuentran varias definiciones de placebo, si bien muchas comparten la idea general que se tiene del mismo, son interesantes por sus matices diferenciales.

Trabajo aceptado: 17 de abril de 2007

Correspondencia: Susana Gabriela Tempone Pérez. Servicio de Anestesiología. Hospital Italiano de Buenos Aires. Argentina. Gascón 450 (C1181ACH). e-mail: susanagabrielaperez@yahoo.com.ar 
Comenzando por la definición de la Real Academia Española, placebo es una "sustancia que, careciendo por sí misma de acción terapéutica, produce algún efecto curativo en el enfermo, si este la recibe convencido de que esa sustancia posee realmente tal acción", es de notar que ésta definición se divide en dos partes, por un lado define al placebo como sustancia que carece de acción terapéutica, pero inmediatamente define la condición del sujeto para que dicha sustancia.

Bugel comenta que a través de la historia de la medicina, los procesos curativos se han explicado de 3 formas generales: a) según los efectos del tratamiento médico establecido; b) debidos al poder sanador de la naturaleza; y c) por el efecto placebo. Establece que el efecto placebo es el tratamiento que no puede ser explicado por la ciencia (5).

Vrhovac B describe que el placebo se puede definir como "un efecto terapéutico, farmacológico o no farmacológico, sobre la condición tratada aún sin tener una acción específica" (3). En el mismo artículo señala que el efecto placebo está especialmente pronunciado en enfermedades donde el dolor es un síntoma importante. La reacción al efecto placebo, según Vrhovac, dependerá también de la personalidad del médico que la receta así como de su actitud y de la personalidad del paciente.

En un trabajo de Papakostas y col comentan que el concepto de placebo ha sufrido dramáticas transformaciones durante el paso del tiempo. Por siglos, los placebos han sido considerados terapias "engañosas" de gran alcance en la población. Sin embargo, desde la mitad del siglo veinte se han incorporado como herramientas utilizadas en la investigación clínica (6).

Kienle y col definen el efecto placebo en forma muy amplia y dicen: "es una acción específica sobre la condición para la cual es dado" (7). En el mismo artículo Kienle y col informan que desde el año 1955 cuando Beecher publicó su obra clásica "El efecto placebo" se ha aceptado generalmente que $35 \%$ de los pacientes pueden ser tratados con placebo. Desde entonces se ha postulado que pueden prolongar la vida, que tienen efectos en la cirugía así como en otras entidades clínicas y que su mecanismo de acción está mediado por endorfinas. La endorfina es un polipéptido producido en el organismo como respuesta a varias situaciones, entre las cuales se encuentra el dolor. Las endorfinas se descubrieron en 1975, posteriormente a la identificación de los receptores específicos para la morfina. Se consideró que el organismo tenía estos receptores para una sustancia endógena (producida dentro del organismo). Ciertamente está relacionada con la génesis de otras emociones, sin embargo, las evidencias señalan que la principal función es en la modulación del dolor.

Margo dice que el efecto de las sustancias inertes sobre el organismo es bien conocido, sin embargo no hay evidencias que nos permitan explicar científicamente que es un placebo y que es el efecto placebo (8). En el mismo trabajo comenta que el rol de los placebos en la medicina moderna está pobremente definido por dos motivos principales; por un lado, por la falta de conocimiento sobre su acción y por otro lado, por las connotaciones negativas que tiene socialmente y para algunos médicos el uso de placebo.

De Deyn utiliza la definición de Shapiro que define al placebo como "cualquier procedimiento terapéutico (o componente del mismo) que es administrado deliberadamente para tener un efecto sobre un síntoma, un síndrome, o un signo pero que es objetivamente una sustancia inerte" (9).
Podemos concluir que en términos generales "el efecto placebo es la modificación inducida de síntomas, signos u otros indicadores, que experimenta el organismo, que no es atribuible al mecanismo de acción específico de una terapéutica, ya sea mecánica, farmacológica, quirúrgica, ambiental o debida a cualquier otra situación curativa". Pero no debemos olvidar que los mecanismos a través de los cuales se lleva a cabo éste efecto aún no han sido esclarecidos.

\section{USO DEL PLACEBO EN MEDICINA}

El uso del placebo es evidente en algunas enfermedades donde el dolor juega un rol importante y en enfermedades psicosomáticas. El grado de la reacción del placebo depende, entre otros factores, de las características del paciente; por ello se los divide como respondedores y no respondedores al placebo; y de la actitud del médico que lo indica (3).

En oncología como lo señala el Dr Pérgola los médicos se toman muchas tribulaciones al recetar un placebo así como el oncólogo tiene muchos problemas al prescribir un medicamento que podría desestabilizar el sistema inmune (4). En el mismo trabajo señala la asociación entre el uso de placebo y dolor, donde "efecto placebo", logra un $30 \%$ de "buenos resultados", mitigando el dolor y/o mejorando el ánimo o la esperanza del enfermo (4).

Como lo menciona Zeller y col desde el punto de vista científico y desde la perspectiva de la medicina basada en la evidencia, no hay una indicación precisa de terapia en base a placebo, excepto algunos casos de patologías asociadas con dolor (10).

Bostrom comenta que hay muchos estudios que han tratado de explicar el efecto del placebo, sin embargo, ha sugerido que actuarían disminuyendo la ansiedad del paciente, o como un mecanismo de respuesta condicionada; también es cierto que quizás el placebo dé respuesta a las expectativas con las que el paciente concurre al médico. Muchas veces hemos escuchado decir a algunos pacientes que su médico no les dio un medicamento y hasta expresan frustración por ello (11).

Hrobjartsson y col realizaron un estudio en humanos, comparando el placebo con la ausencia de tratamiento. Clasificaron a los placebos en 3 tipos: farmacológicos, administraban a los pacientes un remedio no activo; físico, indicándole al paciente actividades que debía realizar en forma controlada por ellos, y psicológicos con terapia. Informan que no encontraron diferencia significativa entre los grupos, por ello, concluyen que fuera del ámbito de la investigación clínica, los placebos no deberían ser utilizados (12).

Tanto las sustancias inertes como activas pueden producir un efecto, originando respuestas que van mas allá de las esperadas para ese producto. Estas observaciones clínicas de las consecuencias de la administración de un placebo comprenden el total de efectos inesperados observados luego de la administración del mismo, y constituyen el denominado efecto placebo (9).

Hay algunas controversias con los placebos que es interesante mencionar: a) si el médico le dice a un paciente que lo que le prescribe es una pastilla de talco, la respuesta placebo que depende en gran medida de las expectativas del paciente, se perderá; b) si el médico le dice al paciente que el placebo que le prescribe es una droga activa, la situación no es ética por falsedad de la información y a la larga será poco eficaz; y 
c) si la enfermedad mejora con placebo significa que parte de la enfermedad está en la "mente" del paciente.

Es importante destacar que el número de consultas de la medicina alternativa supera el número de consultas de los médicos de atención primaria, habría que investigar cual es el papel del efecto placebo en éstas prácticas médicas. El 30\% de los norteamericanos usan terapias alternativas. Se cree que más del 90\% de la medicina alternativa está basada en el efecto placebo.

En medicina clínica no hay claras evidencias que indiquen cuando usar o no un placebo como parte del tratamiento administrado al paciente. Se ha postulado el efecto del mismo en patologías como asma, dolor, migraña, entre otras. Es evidente que la sola presencia del médico frente al paciente ejerce un efecto, y si la administración de una sustancia inerte frente a la ausencia de opción terapéutica refuerza el mismo, debería evaluarse la posibilidad de administrar un placebo, y contemplar estudios científicos mas detallados sobre el tema. Es importante mencionar que no siempre el efecto observado posteriormente a la administración de una sustancia inerte es beneficioso, en el caso de que el mismo sea negativo se lo denomina: efecto nocebo.

\section{USO DEL PLACEBO EN INVESTIGACIÓN CLÍNICA}

En el año 1979 se publica el Informe Belmont (13), el mismo establece cuatro principios ampliados con posteridad, pero que básicamente establecen $(4,13)$ :

1. Respeto a las personas: se divide en dos prerrequisitos morales distintos: el prerrequisito que reconoce la autonomía, y el prerrequisito que requiere la protección de aquellos cuya autonomía está de algún modo disminuida

2. Beneficencia: se entiende en sentido más radical, como una obligación. Dos reglas generales han sido formuladas como expresiones complementarias de los actos de beneficencia entendidos en este sentido: no causar ningún daño, y maximizar los beneficios posibles y disminuir los posibles daños.

3. No maleficencia: principio que podría incluirse en el anterior.

4. Justicia: en el sentido de "equidad en la distribución", o "lo que es merecido".

Como le explica Pérgola, estos principios deben regir el ensayo clínico (4).

Hoy en día se reconoce el fuerte lazo que liga a la investigación científica con la ética, y si un ensayo no está científicamente bien desarrollado automáticamente deja de ser ético.

En investigación clínica los placebos se utilizan deliberadamente para discriminar los efectos farmacológicos de la droga en estudio, de aquellos que no lo son, de ésta forma se pueden separar objetivamente los efectos de la droga estudiada de aquellos que pueden ser de la enfermedad, de su propia evolución, o de otros factores en juego (3). Papakostas y cols. destacan que en la medicina actual el uso de estudios doble ciego, en los cuales un grupo de pacientes reciben tratamiento con la droga en evaluación y el otro sólo recibe placebo, es el diseño de elección para el estudio de nuevos fármacos (6).

En la declaración de Helsinski se establece cuando se puede utilizar el placebo en investigación clínica: "Los posibles beneficios, riesgos, costos y eficacia de todo procedimiento nuevo deben ser evaluados mediante su comparación con los mejores métodos preventivos, diagnósticos y terapéuticos existentes. Ello no excluye que pueda usarse un placebo, o ningún tratamiento, en estudios para los que no hay procedimientos preventivos, diagnósticos o terapéuticos probados". En el año 2001 se agregó a este punto una nota aclaratoria estableciendo que: "Los ensayos con placebo son aceptables éticamente en ciertos casos, incluso si se dispone de una terapia probada y si se cumplen las siguientes condiciones: Cuando por razones metodológicas, científicas y apremiantes, su uso es necesario para determinar la eficacia y la seguridad de un método preventivo, diagnóstico o terapéutico, Cuando se prueba un método preventivo, diagnóstico o terapéutico para una enfermedad de menos importancia que no implique un riesgo adicional, efectos adversos graves o daño irreversible para los pacientes que reciben el placebo" (14).

En investigación clínica el uso del placebo está cuidadosamente indicado, pues diversos problemas éticos surgen del uso del mismo. Siempre nos preguntamos si es ético privar a un porcentaje de los pacientes de un tratamiento concreto, y donde quedan los derechos a un tratamiento en éstas cuestiones. El consentimiento informado, del cual no entraremos en detalle, se transforma en éstos casos en una herramienta esencial del protocolo $(13,14)$. Es por ello que la necesidad de utilizar un estudio controlado con placebo debe ser cuidadosamente evaluado en cada caso en particular.

La Administración de drogas y alimentos (FDA) establece que un protocolo debe ser aprobado por el centro donde se desarrollará la investigación (esto incluye dos aprobaciones, la del Comité de ética y la del de Docencia e Investigación) y por un Comité de Ética Independiente. Cada organismo tiene una reglamentación sobre el uso del placebo que cumple con los criterios del informe Belmont, de la Declaración de Helsisnky y de las Buenas Prácticas Clínicas. Mundialmente se ha aceptado que la única justificación para el uso de placebos en investigación clínica es cuando no existe un tratamiento alternativo, evitando provocar un riesgo adicional con el mismo.

\section{CONCLUSIÓN}

Se han analizado los aspectos clínicos, médicos y éticos del placebo relacionados con la investigación clínica. Éticamente se acepta el uso de placebos en circunstancias como: a) frente a la ausencia de un tratamiento efectivo; b) cuando el tratamiento con placebos es corto; c) cuando no agrega riesgos no relacionados a su patología de base; y d) cuando el consentimiento informado firmado por el paciente ha sido claro al respecto.

\section{Bibliografía}

1. Dr. Pedro M. Polito, "El efecto placebo" publicado en http://www. fmed.uba.ar/depto/farmaco2/place.htm

2. Lilienfeld AM The Fielding H. Garrison Lecture: Ceteris paribus: the evolution of the clinical trial. Bull Hist Med 1982; 56: 1-18.

3. Vrhovac B. Placebo and its importance in medicine. Int J Clin Pharma- col Biopharm 1977; 15: 161-5

4. Pérgola F, Fustinoni JC. El dilema de la terapia del cáncer y la relación oncólogo-paciente. Buenos Aires: Medicina 1998; 58: 314-8.

5. Bugel P. The Many Meanings of Placebo. Forsch Komplementarmed. 1998; 5 (Supl. S1): 23-30. 
6. Papakostas YG, Daras MD. Placebos, placebo effect, and the response to the healing situation: The evolution of a concept. Epilepsia 2001; 42: 1614-25.

7. Kienle GS, Kiene H. Placebo effect and placebo concept: A critical methodological and conceptual analysis of reports on the magnitude of the placebo effect. Altern Ther Health Med 1996; 2: 39-54

8. Margo CE. The placebo effect. Surv Ophthalmol 2000; 44: 364-5.

9. De Deyn, P P; D Hooge, R Placebos in clinical practice and research. J Med Ethics 1996; 22: 140-6.

10. Zeller A, Estlinbaum T. Placebo: An unappreciated factor in medicine. Schweiz Rundsch Med Prax 2002; 91: 1986-91

11. Bostrom H. Placebo-the forgotten drug. Scand J Work Environ Health 1997; 23 (Supl. 3): 53-7.

12. Hrobjartsson A, Gotzsche PC. Is the placebo powerless? An analysis of clinical trials comparing placebo with no treatment. New Engl J Med 2001; 344: 1594-602.

13. El Informe Belmont principios y guías éticos para la protección de los sujetos humanos de investigación comisión nacional para la protección de los sujetos humanos de investigación biomédica y del comportamiento. EE.UU. 18 de abril de 1979.

14. Declaración de Helsinki de la Asociación Médica Mundial Principios éticos para las investigaciones médicas en seres humanos. Adoptada por la $18^{\mathrm{a}}$ Asamblea Médica Mundial Helsinki, Finlandia, junio 1964 y enmendada por la $29^{a}$ Asamblea Médica Mundial Tokio, Japón, octubre $1975,35^{\text {a }}$ Asamblea Médica Mundial Venecia, Italia, octubre 1983, $41^{\text {a }}$ Asamblea Médica Mundial Hong Kong, septiembre 1989, 48 a Asamblea General Somerset West, Sudáfrica, octubre 1996 y la 52 ${ }^{\mathrm{a}}$ Asamblea General Edimburgo, Escocia, octubre 2000. 